\title{
Superselective Embolization of Bilateral Inferior Vesical Arteries for the Management of Radiation-Induced Hemorrhagic Cystitis Refractory to Conventional Methods
}

\author{
Jung Eun Lee, ${ }^{1}$ Jae Hyun Kwon, ${ }^{1,}$ and Jungbum Bae ${ }^{2}$ \\ ${ }^{1}$ Department of Radiology, Ilsan Hospital, Graduate School of Medicine, Dongguk University, Goyang, South Korea \\ ${ }^{2}$ Department of Urology, Ilsan Hospital, Graduate School of Medicine, Dongguk University, Goyang, South Korea \\ "Corresponding author: Jae Hyun Kwon, Department of Radiology, Ilsan Hospital, Graduate School of Medicine, Dongguk University, 27 Dongguk-ro, Siksa-dong, Ilsandong-gu, \\ Goyang-si, Gyeonggi-do, 10326, South Korea. Tel: +82-319617828, Fax: +82-319618281, E-mail: jhkwon17@naver.com
}

Received 2017 December 04; Revised 2018 January 08; Accepted 2018 March 18.

\begin{abstract}
Hemorrhagic cystitis (HC) following radiation therapy of pelvic malignancy is a serious and sometimes life-threatening complication. Various methods including bladder irrigation, fulguration, intravesical instillation, oral or systemic agents, hyperbaric oxygen therapy and surgical methods have been proposed to control hematuria. However, there is no consensus on the optimal therapeutic strategy for radiation-induced HC. We recently performed superselective embolization of the bilateral inferior vesical arteries in a patient with radiation-induced $\mathrm{HC}$ refractory to conventional treatment methods. This technique is effective for achieving the control of refractory hemorrhage and can be considered an option for the treatment of radiation-induced HC.
\end{abstract}

Keywords: Radiotherapy, Hemorrhagic Cystitis, Hematuria, Electrocoagulation, Embolization

\section{Introduction}

Hemorrhagic cystitis (HC) induced by radiotherapy may appear as late as 20 years after radiotherapy for the pelvis, and the symptoms can vary from mild to lifethreatening $(1,2)$. Various treatment methods have been used to treat radiation-induced $\mathrm{HC}$, including fulguration, intravesical instillation, oral agents, hyperbaric oxygen (HBO) therapy and surgical treatments. However, no standard treatment methods are currently established (14). Percutaneous arterial embolotherapy can be a minimally invasive and less morbid option for the treatment of radiation-induced $\mathrm{HC}$ refractory to conventional treatment methods $(5,6)$. We report our experience of a patient with radiation-induced $\mathrm{HC}$ refractory to electrocoagulation and continuous bladder irrigation who was successfully managed with embolization of both inferior vesical arteries superselectively.

\section{Case Presentation}

A 79-year-old man was referred to our department due to intractable gross hematuria for 4 months. He had undergone transurethral resection of the bladder (TURB) for prostatic cancer 3 years previously. The prostatic cancer was found to be stage IV (T4NoMo), and the Gleason grading score was $8(4+4)$ based on histopathologic examination. The prostate specific antigen (PSA) score was 0.93 $\mathrm{ng} / \mathrm{mL}$ (normal range, $0-3.0$ ) at the time of surgery. After undergoing TURB for prostatic cancer in our hospital, the patient received androgen deprivation therapy with leuprolide and bicalutamide for 10 weeks. Additionally, the patient received intensity-modulated radiotherapy for the seminal vesicle and whole pelvis for 10 weeks with a total dose of 78 Grays. The patient had multiple co-morbidities including alcoholic liver cirrhosis, diabetes, hypertension, myocardial infarction and gastrointestinal stromal tumor in the stomach, which had been resected 1 year and 6 months previously.

At the time of admission to the hospital with gross hematuria, cystoscopy revealed engorged vessels in the left side bladder wall and multiple bleeding foci in the bladder neck and prostate fossa. Laboratory findings revealed a white blood cell count of $5570 / \mu \mathrm{L}$, a hemoglobin level of $10.7 \mathrm{~g} / \mathrm{dL}$, a hematocrit of $30.4 \%$, a platelet count of $105,000 / \mu \mathrm{L}$, a prothrombin time international normalized ratio (INR) of 1.2 (0.87 - 1.12), and an activated prothrombin time of $29.4 \mathrm{~s}(27.0$ - 42.0). Gross visual examination of urine revealed turbid and red-colored urine. Microscopic examination of urine sediment revealed many red blood 
cells occupying more than half of the high-power field. At first, transurethral resection (TUR) was performed for the engorged vessel in the left side wall of the urinary bladder and electrocoagulation for bleeding foci of the bladder neck and prostate fossa, followed by continuous irrigation of the urinary bladder with normal saline via a Foley catheter. The gross hematuria decreased shortly after TUR and electrocoagulation for the bleeding foci and continuous irrigation of the bladder with normal saline. However, the patient showed continued gross hematuria and had to be given a transfusion of packed red blood cells due to anemia induced by gross hematuria 1 week after the procedure. Because the gross hematuria continued, the patient had to undergo an additional five sessions of electrocoagulation for multiple bleeding foci of the neck and prostate fossa of the urinary bladder with continuous irrigation of the urinary bladder with normal saline via a Foley catheter for 2 months of hospital stay. The patient had to keep the Foley catheter to irrigate the urinary bladder with normal saline, which made him uncomfortable and prevented him from carrying out activities of everyday life.

The patient was referred to the interventional radiology department for management of intractable gross hematuria despite six sessions of electrocoagulation and saline irrigation. We planned to perform superselective embolization of both inferior vesical arteries corresponding to bleeding foci of the urinary bladder. The right common femoral artery was catheterized after local anesthesia with the insertion of a 5-French vascular sheath. A 5-French catheter (Roberts Uterine Catheter; Cook Incorporated, Bloomington, IN, USA) was introduced into the left internal iliac artery to localize vesical arteries. Subsequently a co-axial 1.9-French microcatheter (Tellus; ASAHI INTECC Co., Ltd., Seto, Japan) was used to cannulate the left inferior vesical artery. Embolization of left inferior vesical arteries was carried out with Gelfoam particles (500 - 700 $\mu \mathrm{m}$, Cali-gel, Hangzhou Alicon Pharm Sci. \& Tec. Co, Ltd., Hangzhou City, Zhejiang, China). After embolization of the left inferior vesical artery, the right inferior vesical artery was selectively embolized with Gelfoam particles in the same manner on the right side (Figure 1). After embolization of both inferior vesical arteries, gross hematuria was resolved gradually and finally stopped 2 weeks after embolization. The patient was discharged without recurrence of gross hematuria 2 weeks after embolization of both inferior vesical arteries. The patient underwent one session of transurethral coagulation due to one episode of gross hematuria. Although the patient occasionally showed a small amount of recurrent gross hematuria or clot passage without clinical significance after the treatment, he is doing well without any serious events 6 months after embolization of both inferior vesical arteries.

\section{Discussion}

External beam radiation therapy for the pelvis is widely used as a curative treatment of prostatic cancer $(2,3)$. Acute symptoms of radiation-induced HC include urinary frequency, urgency, and dysuria, which may be self-limiting and persist up to 3 months after radiation therapy (1-3). Acute lower urinary tract symptoms occurring within 6 months after pelvic radiation therapy develop in up to $42 \%$ of patients (4). Late radiation-induced HC can occur from 6 months up to 20 years after radiation therapy, with a mean latent period of 35 months (1). Approximately $6.5-10 \%$ of patients will develop chronic symptoms related to pelvic radiation therapy (2-4). In the late radiation tissue injury, the small blood vessels of the bladder wall are progressively obliterated, resulting in hypoxia, atrophy, and fibrosis, and subsequently necrosis of the bladder mucosa (1). Thereafter, the fibrosis causes the bladder capacity and compliance to decrease, resulting in the patients presenting with urinary frequency, dysuria, and urgency. Eventually, obliterative endarteritis results in ischemia and ulceration of the mucosa and bleeding (2). The predisposing factors of radiotherapy-related late complications are higher doses of radiation (> $70 \mathrm{~Gy}$ ), total bladder dose and fraction, delivery mode (external beam and/or brachytherapy), concurrent treatments, and larger treatment area $(1,2)$. Hematuria is the main presenting symptom of late radiation-induced $\mathrm{HC}$, and can vary from mild to life threatening (1).

Although radiation-induced HC is a potentially serious complication, which may lead to significant morbidity and an increase in cost with prolonged hospitalization, there is no established consensus on the optimal treatment $(1,3$, 4). Various therapeutic methods have been proposed for treating radiation-induced $\mathrm{HC}$, including oral or intravesical agents, fulguration, HBO therapy, embolization of internal iliac artery, and urinary diversion with or without cystectomy (1-4).

Generally, the initial treatment for radiation-induced HC includes the replacement of intravenous fluid and blood transfusion, if necessary, and bladder irrigation, followed by transurethral catheterization $(2,3)$. All clots should be removed before starting continuous irrigation to prevent over-distension and potential rupture of the bladder (1). Several agents, including formaldehyde, alum, silver nitrate, and carboprost, have been used for intravesical instillation therapy, provoking sterilization, lavage, and arrest of focal bleeding foci (3). The HBO therapy is performed by administering $100 \%$ oxygen in a hyperbaric chamber (3). The HBO therapy promotes the angiogenesis and activity of fibroblasts in damaged tissue by creating conditions in which hemoglobin is completely saturated and oxygen is dissolved to high concentrations in the blood plasma $(1,3)$. The success rate of HBO ther- 

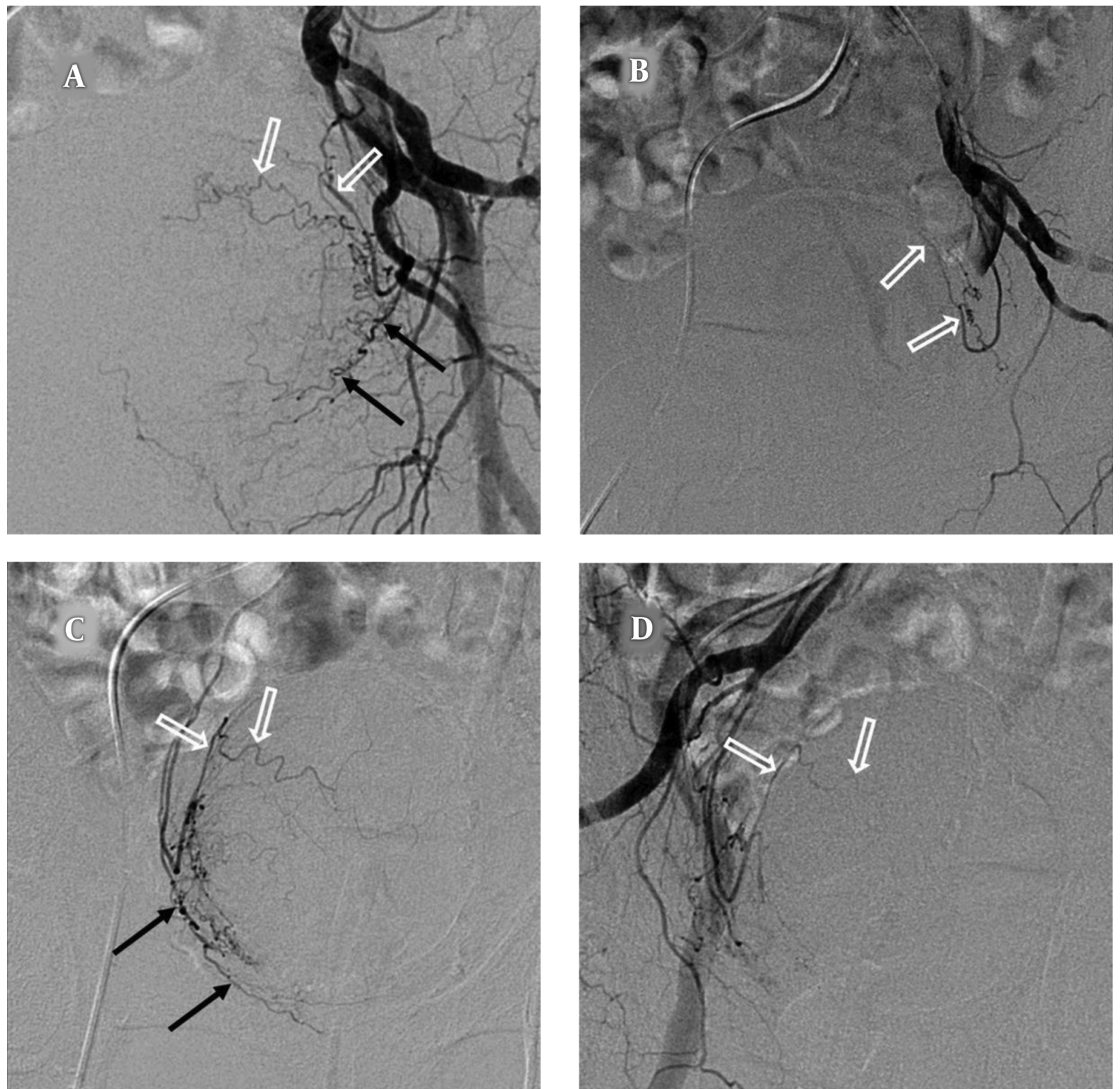

Figure 1. A 79-year-old male patient with radiation-induced hemorrhagic cystitis; A, Angiography of the left internal iliac artery shows the inferior vesical artery (black arrows) and superior vesical artery (open arrows); B, Angiography after superselective embolization of the left inferior vesical artery reveals a preserved superior vesical artery (open arrows) that was still contrast-filled and non-visualization of the embolized inferior vesical artery; C, Angiography of the right vesical artery shows the inferior vesical artery (black arrows) and superior vesical artery (open arrows); D, Angiography after superselective embolization of the right inferior vesical artery reveals a preserved superior vesical artery (open arrows) that was still contrast-filled and non-visualization of embolized inferior vesical artery.

apy for radiation-induced HC, as reported in the literature, ranges from $60 \%$ to $92 \%$ (2). While HBO therapy appears to be an effective treatment method for radiation-induced $\mathrm{HC}$, the difficulties of longer-term administration and access/availability potentially become an obstacle to its more widespread use (1-3).

Cystoscopy with fulguration of bleeding points is an effective treatment for radiation-induced HC that is unre- sponsive to conservative treatments $(1,3)$. Methods of fulguration include electrocoagulation, diathermy, and several types of lasers (1). In this patient, however, electrocoagulation with continuous bladder irrigation was used to treat radiation-induced HC without the improvement of the patient's symptoms during 2 months of hospital admission. Other treatment options besides surgical treatment are superselective embolization of vesical arteries, 
which can be done for patients refractory to electrocoagulation and continuous bladder irrigation. We planned to perform superselective embolization of both inferior vesical arteries supplying the bladder neck portion corresponding to bleeding foci. Superior vesical arteries were preserved to prevent ischemic complications, such as bladder necrosis. Superselective embolization of inferior vesical arteries can also prevent ischemic complications, such as gluteal pain or necrosis, genital injury, or tissue necrosis $(5,6)$.

Embolizations of the entire internal iliac artery or arteries to treat radiation-induced HC were reported with successful results; however, serious complications following embolization of the bilateral internal arteries have been reported $(5,7,8)$. Superselective vesical artery embolization has been used as a therapeutic strategy of HC secondary to radiation therapy, trauma, and tumors without ischemic complications $(5,6,9,10)$. The patient in this study showed gradual decline of hematuria after superselective embolization of bilateral inferior vesical arteries and eventual stoppage of hematuria without ischemic complications. The patient did not experience serious recurrent gross hematuria during the 6-month follow-up period and is doing well, although he showed a small amount of gross hematuria and clot passage without clinical significance. However, longer follow-up periods are required with a larger patient number to establish the long-term efficacy of the superselective embolization of vesical arteries in patients with radiation-induced HC refractory to other treatment modalities $(5,6)$. Surgical treatment, including urinary diversion with or without cystectomy, has been used, but it would be considered a last resort because of high morbidity and mortality (3).

In conclusion, superselective embolization of vesical arteries can not only control radiation-induced hemorrhage, but also minimize the ischemic complications, such as bladder necrosis, gluteal pain or necrosis, or tissue necrosis. In patients with radiation-induced $\mathrm{HC}$ refractory to conventional treatments, superselective embolization of vesical arteries should be considered one of the treatment methods.

\section{Footnotes}

Authors' Contributions: Study concept and design: Jae Hyun Kwon; acquisition of the data: Jung Eun Lee; analysis and interpretation of the data: Jae Hyun Kwon, Jungbum Bae; drafting of the manuscript: Jung Eun Lee, Jae Hyun Kwon; critical revision of the manuscript for important intellectual content: Jae Hyun Kwon, Jungbum Bae; and study supervision: Jae Hyun Kwon.

Financial Disclosure: All authors declare that they have no conflicts of interest.

Funding/Support: None declared.

\section{References}

1. Zwaans BM, Nicolai HG, Chancellor MB, Lamb LE. Challenges and Opportunities in Radiation-induced Hemorrhagic Cystitis. Rev Urol. 2016;18(2):57-65. doi: 10.3909/riu0700. [PubMed: 27601964]. [PubMed Central: PMC5010626].

2. Alesawi AM, El-Hakim A, Zorn KC, Saad F. Radiation-induced hemorrhagic cystitis. Curr Opin Support Palliat Care. 2014;8(3):235-40. doi: 10.1097/SPC.0000000000000073. [PubMed: 25004179].

3. Smit SG, Heyns CF. Management of radiation cystitis. Nat Rev Urol. 2010;7(4):206-14. doi: 10.1038/nrurol.2010.23. [PubMed: 20212517].

4. Crew JP, Jephcott CR, Reynard JM. Radiation-induced haemorrhagic cystitis. Eur Urol. 2001;40(2):111-23. doi: 10.1159/000049760. [PubMed: 11528186].

5. Cho CL, Lai MH, So HS, Kwok KK, Chan JC, Velayudhan V. Superselective embolisation of bilateral superior vesical arteries for management of haemorrhagic cystitis. Hong Kong Med J. 2008;14(6):485-8. [PubMed: 19060349].

6. De Berardinis E, Vicini P, Salvatori F, Sciarra A, Gentile V, Di Silverio F. Superselective embolization of bladder arteries in the treatment of intractable bladder haemorrhage. Int J Urol. 2005;12(5):503-5. doi: 10.1111/j.1442-2042.2005.01074.x. [PubMed: 15948754].

7. Braf ZF, Koontz WW. Gangrene of bladder: complication of hypogastric artery embolization. Urology. 1977;9(6):670-1. doi: 10.1016/0090 4295(77)90319-3.

8. Duff C, Simmen HP, Brunner U, Bauer E, Turina M. Gluteal necrosis after acute ischemia of the internal iliac arteries.Vasa.1990;19(3):252-6. [PubMed: 2238821].

9. Hayes MC, Wilson NM, Page A, Harrison GS. Selective embolization of bladder tumours. Br J Urol.1996;78(2):311-2. [PubMed: 8813940].

10. Gine E, Rovira M, Real I, Burrel M, Montana J, Carreras E, et al. Successful treatment of severe hemorrhagic cystitis after hemopoietic cell transplantation by selective embolization of the vesical arteries. Bone Marrow Transplant. 2003;31(10):923-5. doi: 10.1038/sj.bmt.1703954. [PubMed: 12748670]. 